Document downloaded from:

http://hdl.handle.net/10251/107327

This paper must be cited as:

Arnal Pastor, MP.; Comín-Cebrián, S.; Martínez-Ramos, C.; Monleón Pradas, M.; Vallés Lluch, A. (2016). Hydrophilic surface modification of acrylate-based biomaterials. Journal of Biomaterials Applications. 30(9):1429-1441. doi:10.1177/0885328215627414

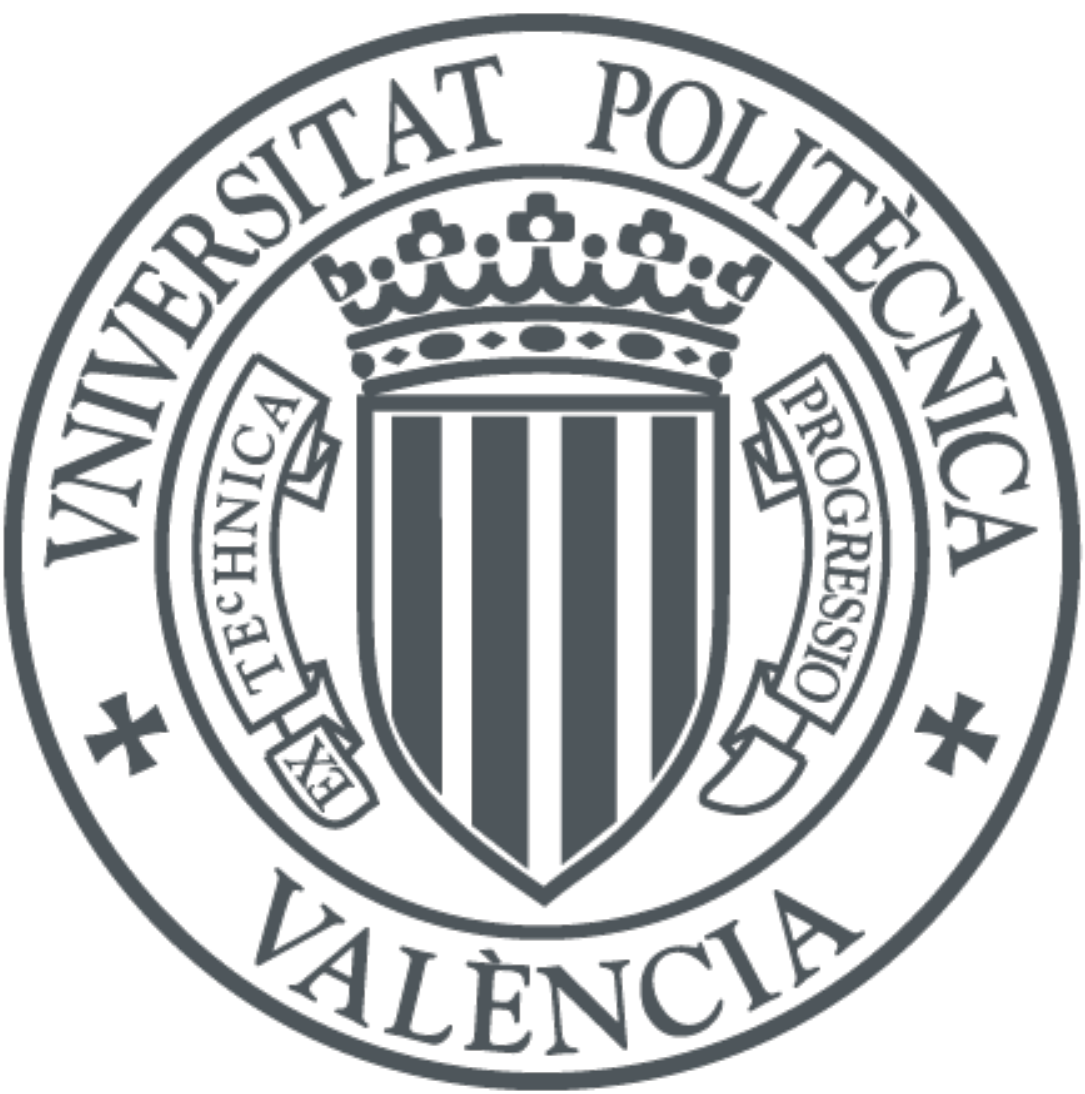

The final publication is available at

https://doi.org/10.1177/0885328215627414

Copyright SAGE Publications

Additional Information 


\title{
HYDROPHILIC SURFACE MODIFICATION OF ACRYLATE-BASED BIOMATERIALS
}

M. Arnal-Pastor ${ }^{1}$, S. Comín-Cebrián ${ }^{1}$, C. Martínez-Ramos ${ }^{1}$, M. Monleón Pradas ${ }^{1,2}$, A. Vallés-Lluch, ${ }^{1, *}$

${ }^{1}$ Center for Biomaterials and Tissue Engineering, Universitat Politècnica de València, C. de Vera s/n, 46022, Valencia, Spain

${ }^{2}$ Networking Research Center on Bioengineering, Biomaterials and Nanomedicine, Valencia, Spain *Corresponding author. Tel.: +34963877000; fax: +34963877276. E-mail: avalles@ter.upv.es

\begin{abstract}
Acrylic polymers have proved to be excellent with regard to cell adhesion, colonization and survival, in vitro and in vivo. Highly ordered and regular pore structures thereof can be produced with the help of polyamide templates, which are removed with nitric acid. This treatment converts a fraction of the ethyl acrylate side groups into acrylic acid, turning poly(ethyl acrylate) (PEA) scaffolds into a more hydrophilic and $\mathrm{pH}$ sensitive substrate, while its good biological performance remains intact. To quantify the extent of such a modification, and be able to characterize the degree of hydrophilicity of PEA, PEA was treated with acid for different times (4, 9 and 17 days), and compared with poly(acrylic acid) (PAAc) and a 90/10 \%wt. EA/AAc copolymer (P(EA-co-AAc)). The biological performance was also assessed for samples immersed in acid up to 4 days and the copolymer, and it was found that the incorporation of acidic units on the material surface was not prejudicial for cells. This surface modification of 3D porous hydrophobic scaffolds makes easier the wetting with culture medium and aqueous
\end{abstract}


solutions in general, and thus represents an advantage in the manageability of the scaffolds.

Keywords: scaffold, nitric acid, ethyl acrylate, acrylic acid, surface modification 


\section{Introduction}

Poly(ethyl acrylate) (PEA) is a polymer with a low hydrophilicity, elastomeric at body temperature. Its mechanical properties are close to those of soft biological tissues. PEA has shown a very good biological performance in vitro [1-7] and in vivo [8].

Different porous architectures thereof can be produced by employing a porogen template with the negative of the pore structure, which after the polymerization is removed by dissolution: scaffolds with interconnected spherical pores $[1,8,9]$, unidirectional cylindrical channels [10], or orthogonally intersecting cylindrical channels $[8,10,11]$. For the latter, a porogenic template made of polyamide-6 (PA-6) sintered fabrics is used, which was removed by dissolution in nitric acid. The present paper focuses on the effects of this production step, which involves immersion of the polymer in nitric acid for a time long enough to dissolve the porogen, on the physicochemical and biological properties of the PEA matrix. The use of acid in the production of these scaffolds becomes necessary because this particular pore architecture is obtained by sintering PA-6 fabrics, which are easily available commercially, and acids are generally the sole good solvents for many semicrystalline polymers such as PA-6. Acids dissolve the fabrics template, but respect the insoluble PEA network. Moreover, this process simultaneously improves the polymer hydrophilicity, enhancing the wettability and cell collonization of the scaffold.

It is well known that the presence of certain chemical groups on the surface of the substrates can modulate their biological outcome [12-14]. In particular, the presence of acid groups improves wettability, which can facilitate the seeding of porous materials 
[15], but also the presence of surface charges are reported to affect the deposition of ECM-proteins [16-18] that would translate into a better cell-material interaction or differentiation for certain cell lines [5, 19, 20]. Indeed, the preconditioning of hydrophobic PEA scaffolds with a hydrogel has been found to improve significantly cell seeding and migration to colonize them homogeneously [21-23].

To the best of our knowledge, there is no previous systematic characterization of the nitric acid effect on PEA. In the present study PEA was prepared and exposed to an acid treatment of the sort it would suffer during the typical preparation steps of the abovementioned type of scaffold, as described in [10, 11, 24, 25]. EA being the ethyl ester of acrylic acid, it was hypothesized that the effect the nitric acid has on PEA was the hydrolysis of a number of its side chains transforming ethyl acrylate units into acrylic acid and giving rise to a random copolymer containing EA and AAc units, thus reverting the process of acid-catalysed esterification of acrylic acid. In order to prove this hypothesis, acid-treated films were compared with untreated PEA, poly(acrylic acid) (PAAc) and a P(EA-co-AAc) copolymer. The effect of the acid treatment on the biological performance of the materials was evaluated in vitro, and compared with that of untreated PEA, to complete the characterization.

\section{Materials and methods}

\subsection{Materials}

PEA scaffolds with interconnected cylindrical orthogonal pores were obtained following the procedure described in [25]. Briefly, porogenic templates were previously obtained by sintering 8 layers of nylon fabrics with a nominal thread diameter of 150 $\mu \mathrm{m}$ and a mesh opening of $300 \mu \mathrm{m}$ (SAATI S.A.). The reactant mixture, consisting of ethyl acrylate (99\%, Sigma-Aldrich), 0.1 wt.\% of azo-bis-isobutyronitrile (99\%, 
Fluka), AZBN, as thermal initiator, and 2 wt\% of ethyleneglycol dimethacrylate (98\%, Sigma-Aldrich) as cross-linker, was injected in a porogen template, polymerized for 24 $\mathrm{h}$ at $60^{\circ} \mathrm{C}$, and postpolymerized for $24 \mathrm{~h}$ more in an oven at $90^{\circ} \mathrm{C}$. Next, the nylon templates were removed by dissolution in nitric acid (30\%, Aldrich), repeatedly changed, during 4 days to dissolve the nylon template. Then, scaffolds were washed in boiling water for $16 \mathrm{~h}$ (the water was changed every 8 hours) to remove nitric acid traces. The scaffolds obtained were cut, dried under vacuum and observed by scanning electron microscopy.

PEA films were obtained by UV polymerization of reactant ethyl acrylate monomer (EA; 99\%, Sigma-Aldrich), using 1\% of benzoin as initiator (Scharlab) and 2\% of ethyleneglycol dimethacrylate (98\%, Sigma-Aldrich) as crosslinker. The monomeric mixture was stirred during 15 min, injected in a glass flat mould, polymerized for $8 \mathrm{~h}$ in an UV oven and post-polymerized for $24 \mathrm{~h}$ at $90^{\circ} \mathrm{C}$. To remove any unreacted species, samples were washed in boiling ethanol (EtOH; 99\%, Scharlab) during $24 \mathrm{~h}$ with renewal every 8 h. Analogously, PAAc films were obtained from acrylic acid monomer (99\%, Sigma-Aldrich). The same procedure was followed to obtain EA-co-AAc copolymers in a 90:10 weight proportion. After the polymerization and washing steps, 1 mm-thick samples were cut either in $\left(0.5 \times 2.5 \mathrm{~cm}^{2}\right)$ bars or punched as discs of 5,7 or $10 \mathrm{~mm}$ diameter. PEA samples were immersed in a $\mathrm{HNO}_{3}(65 \%$ extra pure, Scharlab)/water 1/1 vol. solution and placed in a shaker for 4 (PEA-4d), 8 (PEA-9d) or 17 days (PEA-17d). At withdrawal, samples were dried with a filter paper and then rinsed for $16 \mathrm{~h}$ in boiling water (with a change after $8 \mathrm{~h}$ ). Next, they were left overnight in fresh water and dried thoroughly. 


\subsection{Methods}

\subsubsection{Scanning electron microscopy (SEM)}

The scaffolds morphology was characterized with a SEM device (Hitachi S-4800) at 10 $\mathrm{kV}$. Both samples’ surfaces and inner sections were observed, after sputter-coating with gold.

\subsubsection{Fourier Transformed infrared spectroscopy (FTIR)}

A Thermo Nicolet Nexus FTIR (Thermo Fischer Scientific Inc., Waltham, MA, USA) spectrometer was employed to determine the surface composition of the samples before and after acid treatments. Spectra were obtained in the ATR mode, with a resolution of $8 \mathrm{~cm}^{-1}$, between 500 and $4000 \mathrm{~cm}^{-1}$ as the average of 68 scans.

\subsubsection{Swelling kinetics experiment}

Samples of the treated and non-treated materials were swollen to equilibrium by immersion in different media to study their $\mathrm{pH}$-sensitivity in terms of the degree of swelling. These media were pure water, a $0.2 \mathrm{M} \mathrm{NaOH}$ aqueous solution, and aqueous solutions with constant ionic strength but different $\mathrm{pH}$ (prepared as described in [26]): $\mathrm{Na}_{2} \mathrm{HPO}_{4}, \mathrm{H}_{3} \mathrm{C}_{6} \mathrm{H}_{5} \mathrm{O}_{7}$ and $\mathrm{KCl}$ were used to obtain solutions with ionic strength of 1.0 $\mathrm{M}$ and $\mathrm{pH}$ of 2, 4.6 and 8, respectively. Samples dried under vacuum were weighed before immersion and at selected time points. After withdrawal, the liquid remaining on the surface was carefully removed prior to weighing. The water uptake was calculated in triplicate as: $W C=\frac{W_{t}-W_{0}}{W_{0}}$, where $W C$ is the water content, $W_{t}$ is the weight of the disc swollen at time $t$ and $W_{0}$ is the weight of the dry disc. The equilibrium water content, $E W C$, is the attained final constant value of $W C$. 


\subsubsection{Density measurements}

Density of the materials was determined with a Mettler Toledo AE-240 balance combined with a density accessory kit through Archimedes’ principle. Samples were weighed in air and in n-octane (95\%, Fluka), and their densities were calculated in triplicate as: $\rho=\frac{\rho_{N-o c t} \cdot W_{\text {air }}}{W_{\text {air }}-W_{N-o c t}}$, where $\rho_{N-o c t}$ is the density of n-octane $\left(0.703 \mathrm{~g} / \mathrm{cm}^{3}\right)$, $W_{\text {air }}$ is the weight of the disc in air and $W_{N-o c t}$ is the weight of the disc immersed in noctane.

\subsubsection{Differential scanning calorimetry (DSC)}

DSC measurements were carried out in a Metter Toledo DSC823e (Columbus, OH, USA) device, to determine the thermal properties of the samples. Specimens were heated in $30 \mu \mathrm{L}$ pierced pans from $-100^{\circ} \mathrm{C}$ to $200^{\circ} \mathrm{C}$ at $10^{\circ} \mathrm{C} / \mathrm{min}$, with a nitrogen flux of $60 \mathrm{~mL} / \mathrm{min}$. The glass transition temperature $\left(T_{g}\right)$ was determined as the midpoint, and the heat capacity increase $\left(\Delta C_{p}\right)$ was obtained for that $T_{g}$.

\subsubsection{Dynamic-mechanical thermal analysis (DMTA)}

DMTA measurements were performed in a Seiko DMS210 instrument (Seiko Instruments Inc., Chiba, Japan) to determine the influence of the acid on the mechanical properties of PEA. Measurements were done from $-60^{\circ} \mathrm{C}$ to $180^{\circ} \mathrm{C}$ at $2^{\circ} \mathrm{C} / \mathrm{min}$, at $1 \mathrm{~Hz}$ in the tensile mode. Samples were rectangular, $5 \mathrm{~mm}$ wide.

\subsubsection{Contact angle measurements}

The contact angle of $3 \mu \mathrm{L}$ drops of extra pure water on the surface of the materials was determined in the sessile-drop mode with a Dataphysics OCA instrument (Filderstadt, 
Germany). Samples were characterized in their dry state and after swelling in water for $48 \mathrm{~h}$, with 10 water drops each.

\subsubsection{Thermogravimetric analysis (TGA)}

TGA scans were conducted in a TA-SDT Q600 device (TA Instruments, New Castle, DE, USA), from 35 to $1000^{\circ} \mathrm{C}$ at $10^{\circ} \mathrm{C} / \mathrm{min}$ and with a $50 \mathrm{~mL} / \mathrm{min}$ nitrogen flux. $10 \mathrm{mg}$ samples were dried under vacuum and $60^{\circ} \mathrm{C}$ for $24 \mathrm{~h}$ before measurements.

\subsubsection{Toluidine blue staining}

A colorimetric characterization of the nitric acid effect was carried out by immersing discs in a $0.1 \%$ toluidine blue (Sigma-Aldrich) aqueous solution for $30 \mathrm{~s}$, followed by a gentle rinse with distilled water to remove the excess of staining.

\subsubsection{Sterilization and conditioning}

Prior to cell culture, discs were washed for $30 \mathrm{~min}$ in an EtOH/ $\mathrm{H}_{2} \mathrm{O} 70 / 30$ mixture in an ultrasound bath (Bandelin Sonorex Digitec). Next, they were rinsed with fresh $\mathrm{EtOH} / \mathrm{H}_{2} \mathrm{O}$ and dried thoroughly. Samples were then sterilized with UV light followed by several DPBS (Dulbecco’s Phosphate Buffered Solution, Sigma) rinses, and kept overnight in fresh DPBS before fibroblasts culture, or in culture medium containing foetal bovine serum (FBS) for cultures with human umbilical vein endothelial cells (HUVECs).

\subsubsection{Cell culture}

L929 mouse fibroblasts (C34/An connective tissue, Sigma Aldrich) in their 18th passage and HUVECs (Gibco) in their 6th passage were used. Fibroblasts were cultured 
with DMEM 4.5g/l glucose (Gibco) containing glutamine (Invitrogen), 10\% of FBS (Invitrogen) and 1\% of penicillin/streptomycin (P/S; Life technology), until confluence. Then, cells were trypsinized with trypsin-EDTA (Invitrogen) for $3 \mathrm{~min}$ at $37^{\circ} \mathrm{C}$, collected and centrifuged at $1000 \mathrm{rpm}$ for $5 \mathrm{~min}$, re-suspended, counted and diluted to the desired cell density. Cells were seeded on 5 or $7 \mathrm{~mm}$ diameter samples as 20 or 40 $\mu \mathrm{L}$ droplets, each one containing 5000 or 9800 cells, respectively. Bidimensional continuous samples, rather than tridimensional scaffolds, were used in cultures as simpler models, so as to avoid cells loss while performing cuts, stainings and rinsings and reveal more clearly differences in the biological performance of the different chemical compositions. The samples were incubated for $30 \mathrm{~min}$, to allow cells to attach before filling the wells with 180 or $400 \mu \mathrm{L}$ of fresh medium, respectively. The medium was changed every day.

HUVECs were cultured with Medium 200 (Invitrogen) supplemented with LSGS (low serum growth supplement, Invitrogen) and gentamicin (Gibco) and P/S until confluence. Then, cells were trypsinized, collected and centrifuged at $180 \mathrm{~g}$ for $7 \mathrm{~min}$. Suspensions of the desired densities were prepared: $20 \mu \mathrm{L}$ droplets containing 7700 cells for $5 \mathrm{~mm}$ diameter samples and 15000 cells in $40 \mu \mathrm{L}$ droplets for the $7 \mathrm{~mm}$ diameter discs. 30 min after seeding, the medium was completed and renewed every day.

\subsubsection{Cell viability assay}

After 6 h, 1, 3 and 5 days of culture MTS assays ((4,5-dimethylthiazol-2-yl)-5-(3 carboxymethoxyphenyl)-2-(4-sulfophenyl)-2H-tetrazolium, Cell titer 96 Aqueous One Solution cell proliferation assay Promega) were performed to study the seeding efficiency and the cell proliferation rate, 3 replicates per time. Briefly, the samples were 
rinsed with DPBS and moved to a new 96-well plate. $200 \mu \mathrm{L}$ of the MTS/phenol redfree medium mixture was added to each well and incubated in the dark. After 3 h, 100 $\mu \mathrm{L}$ aliquots were placed in new wells and their absorbance was measured in a Victor Multilabel Counter 1420 spectrophotometer (Perkin Elmer, Waltham, MA; USA) at 490 nm. Cells cultured directly in polystyrene wells (or on PEA discs in the case of HUVECs) were employed as positive controls. MTS reactant solution incubated in wells with acellular materials worked as negative control.

\subsubsection{Haematoxylin and eosin staining}

At the selected time points samples were fixed with formaldehyde as previously described. Before staining, they were rinsed with distilled water for 3 min followed by other $3 \mathrm{~min}$ in tap water. Samples were next soaked in a haematoxylin solution according to Mayer (Sigma-Aldrich) and incubated for $10 \mathrm{~min}$ at RT, followed by the addition of a bluing solution (tap water, $\mathrm{NaHCO}_{3} 1 \% \mathrm{w} / \mathrm{v}$ (Fluka) and $\mathrm{MgSO}_{4} 0.0667 \%$ w/v (Scharlab) for 90 s. Once removed, type B eosin (Sigma) was added and incubated for 10 min at RT. Finally, samples were rinsed with tap water, mounted with a glycerol (Sigma)/water 85/15\% vol. mixture, coverslipped and observed with a petrographic microscope (Nikon).

\subsubsection{Statistical analysis}

Results are expressed as mean \pm standard deviation from at least three replicates. Data were analysed pair wise with an ANOVA test with Statgraphics Centurion XVI.I. Results are statistically significant unless noted otherwise, except for the MTS results that are explained in detail in the figure caption. Significance was assigned at p-values $<$ 0.05 . 


\section{Results}

\subsection{Morphological characterization}

Interconnected cylindrical orthogonal pores can be clearly observed in a cross section image of the PEA scaffold (Fig. 1-A), as well as several layers (8) of cylindrical crossed pores in parallel and perpendicular planes (Fig. 1-B). The bulk porosity of the scaffolds was $76.4 \pm 6.1 \%$. They were flexible and with pore diameters $(149.08 \pm 17.02 \mu \mathrm{m})$ excellent for cell colonization.

\subsection{Physico-chemical and mechanical characterization}

The FTIR spectra evidence changes occurred in the chemical structure of PEA by the $\mathrm{HNO}_{3}$ treatments (Fig. 2-A). As expected, the $3200 \mathrm{~cm}^{-1}$ wide band (characteristic for the hydroxyl groups) appears for all acid-treated acrylates but not for the untreated PEA (highlighted in the Figure). And in the particular case of PEA-17d the $3500 \mathrm{~cm}^{-1}$ band is present as well (pointed out in the Figure). The PAAc spectrum exhibits both 3500 and $3200 \mathrm{~cm}^{-1}$ bands. In the P(EA-co-AAc) spectrum, only the $3200 \mathrm{~cm}^{-1}$ band is shown.

The symmetric and asymmetric stretches of $\mathrm{CH}_{\mathrm{x}}\left(\mathrm{CH}_{2}, \mathrm{CH}_{3}\right)$ groups appear as peaks in the 2962 and $2888 \mathrm{~cm}^{-1}$ region for all materials. For the PAAc, as for the PEA-17d although more moderately, another wide band is seen in the 2800 to $2500 \mathrm{~cm}^{-1}$ region (pointed out in the Figure), which could be attributed to the carboxylic acid group. This band was not evident for the copolymer.

The $1715 \mathrm{~cm}^{-1}$ peak is present and very well defined for all studied materials, since it is characteristic for carbonyl bonds in the carboxyl group. For the PEA-9d and PEA-17d, a 
slight shift of this peak towards lower wavenumbers and a new one at $1600 \mathrm{~cm}^{-1}$ are perceived. In the particular case of the PAAc, the peak shift is more evident and the new peak is broader.

Samples either untreated or treated for 4 days with nitric acid show a slightly different behaviour in water, but a dramatically different behaviour when swollen in a basic solution (Fig. 3-A) in a preliminary assay. For untreated PEA, there are no significant differences in the water content when swollen in water or in the $\mathrm{NaOH}$ solution; moreover, the swelling in $\mathrm{NaOH}(\mathrm{aq})$ did not increase significantly from $1 \mathrm{~h}$ on. In contrast, the swelling of acid-treated PEA changed from a WC around 16\% after $1 \mathrm{~h}$ up to $130 \%$ after $24 \mathrm{~h}$. When swollen in water, these samples had a $W C$ after 24 h only slightly higher than the untreated ones: $2.78 \%$ vs. $1.14 \%$.

The effect of the duration of the acid treatment on the swelling degree was further characterized by using media with different $\mathrm{pH}$ but constant ionic strength. The goal of these tests were to reveal the chemical modifications occurred during the preparation of the materials. These conditions are not, thus, representative of physiological media. In physiological media, the swelling of each network will vary as a function of $\mathrm{pH}$, ionic strength and temperature, and can be somewhat tailored with its crosslinking degree. When the samples were swollen at $\mathrm{pH} 2$ (Fig. 3-B), the EWC increased slightly with the acid-treatment: $1 \%$ for untreated PEA up to $5 \%$ for PEA-17d. The PEA-9d samples showed quasi-identical values to P(EA-co-AAc). No significant differences between the EWC of the copolymer and PEA treated for 4 and 9 days were observed. The EWC attained by PAAc was much higher (50\%), and P(EA-co-AAc) (2.5\%) appeared to be more hydrophilic than PEA but was far from the values of PAAc. Similar results and 
values were obtained for $\mathrm{pH}$ 4.6. However, when the materials were swollen in a basic medium, i.e. $\mathrm{pH}$ 8, their behaviour changed dramatically except for the untreated PEA, which maintained an EWC around 1\%. The PEA-4d nearly triples its $E W C$ at this $\mathrm{pH}$ reaching a value around 6\%, PEA-9d reached an EWC of nearly $60 \%$, and $240 \%$ was the value attained by PEA-17d. Much higher values were obtained for PAAc (650\%), and $\mathrm{P}(\mathrm{EA}-\mathrm{Co}-\mathrm{AAc})$ reached an intermediate $60 \%$.

The swelling of PEA was independent of the medium $\mathrm{pH}$, with a moderate value of EWC. For the acid-treated samples, as for the copolymer and PAAc, there were no remarkable differences between the $E W C$ attained at $\mathrm{pH}=2$ and at 4.6 , but at $\mathrm{pH}=8$ these materials showed a hydrophilic behaviour, which was proportional to the immersion time for the treated series or to the fraction of carboxyl lateral groups for the reference materials. Interestingly, the PEA-9d and the copolymer swelled at all $\mathrm{pH}$ values following the same trend and attaining a similar EWC.

The density was also sensitive to the nitric acid exposure. The longer the treatment, the greater the density of the resulting material (Fig. 4-A), changing gradually from 1.134 $\mathrm{g} / \mathrm{cm}^{3}$ for untreated PEA up to $1.180 \mathrm{~g} / \mathrm{cm}^{3}$ for PEA-17d. The densities of PEA-4d and PEA-9d were closer to that of the copolymer $\left(1.158 \mathrm{~g} / \mathrm{cm}^{3}\right)$; in fact, no statistically significant difference was observed between PEA-9d and the copolymer. As expected, PAAc exhibited a much higher density, $1.289 \mathrm{~g} / \mathrm{cm}^{3}$.

A displacement of the glass transition towards higher temperatures with the duration of the acid treatment was observed, shifting from 260K for PEA up to 295.4K for PEA17d (Fig. 5). The DSC thermogram of P(EA-Co-AAc) is intermediate to those of PEA- 
4d and PEA-9d, its glass transition temperature (271.21 K) lying between the respective values for these samples. By contrast, PEA-17d behaved similarly to PAAc, although this last's $T_{g}$ was a bit lower, $294.14 \mathrm{~K}$.

The specific heat capacity jump at the glass transition $\left(\Delta c_{p}\right)$ slightly increased with the time of acid treatment, from $0.39 \mathrm{~J} / \mathrm{gK}$ for PEA up to $0.453 \mathrm{~J} / \mathrm{gK}$ for PEA-17d. Again, the PAAc value was the highest $(0.769 \mathrm{~J} / \mathrm{gK})$, and that of the copolymer $(0.423 \mathrm{~J} / \mathrm{gK})$ was of the order of acid treated PEA.

The acid treatments did also influence the viscoelastic behaviour, Fig. 6. At temperatures below $0^{\circ} \mathrm{C}$, all materials displayed a high storage modulus $\left(10^{9}-10^{10} \mathrm{~Pa}\right)$ typical of glassy polymers. The PEA main relaxation was observed in the interval from $-10^{\circ} \mathrm{C}$ to $30^{\circ} \mathrm{C}$; it was shifted to higher temperatures, in the range from -5 to $40^{\circ} \mathrm{C}$ in $\mathrm{P}(\mathrm{EA}-\mathrm{co}-\mathrm{AAc})$ and much higher in PAAc, from $15^{\circ} \mathrm{C}$ to $60^{\circ} \mathrm{C}$. In the case of the acidtreated polymers, the relaxation shifted from that of PEA towards that of P(EA-co-AAc) for PEA-4d, to greater temperatures for PEA-9d and up to values close to those of PAAc for PEA-17d. After this relaxation, the elastic modulus was in all cases in the order of MPa, typical of an elastomer.

As the inset in Fig. 6-B shows, the temperature at which $\tan \delta$ achieves its maximum value $\left(T_{\alpha}\right)$ increases with the duration of the acid treatment. The maximum for P(EAco-AAc) is located between those for PEA-4d and PEA-9d, and that of PAAc is the highest and close to that of the PEA-17d. 
The water contact angle on the surface was also affected by the chemical modifications occurring in the PEA matrix, but was revealed only when the polyacrylates were in their swollen state. Dry acid-treated PEA discs exhibited a slightly lower contact angle than untreated PEA, indicating that after the acid exposure the PEA becomes more wettable (Fig. 7-A). As expected from their chemistry, that includes lateral acid groups, both $\mathrm{P}(\mathrm{EA}-\mathrm{co}-\mathrm{AAc})$ and PAAc exhibited low contact angles, even below those of the treated samples. Surprisingly, despite only $10 \%$ wt of the monomeric units in P(EA-co-AAc) contain an acid group, its surface appeared to be more wettable than PAAc, whose units, all of them, contain such group. The differences between untreated and acid-treated PEA and PAAc were not statistically significant. However, when the contact angles were measured after a previous swelling (Fig. 7-B), the results were different. The contact angle on swollen PEA was still high but somewhat lower than in its dry state, and those of the treated samples decreased with the immersion time in acid up to 9 days. After swelling, the average contact angle of PAAc was lower than that of P(EA-coAAc), as expected given their composition. PEA-4d behaved as P(EA-co-AAc) and PEA-9d had a contact angle close to that of PAAc (there were no statistically significant differences between them). Swollen PEA-17d showed again an anomalous behaviour.

The thermal degradation of PEA is also affected by the acid treatment (Fig. 4-B): the onset of the weight loss is shifted toward lower temperatures and the main thermal degradation of PEA, which occurs between $325^{\circ} \mathrm{C}$ and $425^{\circ} \mathrm{C}$, gradually changes with the duration of the acid treatment up to $260^{\circ} \mathrm{C}-440^{\circ} \mathrm{C}$ for PEA-17d. Once more, PEA-4d and PEA-9d exhibited an intermediate behaviour between those of PEA and the copolymer, undergoing the main thermal degradation in the $295^{\circ} \mathrm{C}-440^{\circ} \mathrm{C}$ interval. The weight of the solid residue slightly increased with the acid treatment time, from $3 \%$ for 
PEA up to $5.4 \%$ for PEA-17d. Although PEA-17d shows a decomposition thermogram not dissimilar to that of P(EA-Co-AAc), its solid residue coincides with that of PAAc.

After staining with toluidine blue, samples were gradually bluer with either the treatment time in nitric acid (Fig 7. A-D) or the fraction of acrylic acid groups (Fig 7. E, F). Untreated PEA acquired a light purplish shade. The colouring of PEA-4d and PEA9d was very similar to that of $\mathrm{P}(\mathrm{EA}-\mathrm{co}-\mathrm{AAc})$ and already in the blue tonality; while PAAc was the most stained sample.

\subsection{Biological experiments}

The MTS assay after the fibroblasts culture (Fig. 8) showed no statistically significant differences among the different materials and positive control, regarding the initial adhesion. In all cases (except for $\mathrm{P}(\mathrm{EA}-\mathrm{co}-\mathrm{AAc}$ ) at day 1), there is a significant difference between the acellular control and the cellular samples indicating that cells attached to the substrates and are viable at longer times. In general, untreated PEA exhibited a better behaviour as compared with the other polymers, and exhibited a closer behaviour to the control (plate well), increasing the absorbance with time. In the case of PEA-4d and P(EA-co-AAc), the absorbance increase was more moderated. Since PEA reached absorbance values close to those of the positive control in fibroblasts culture, in the following HUVECs culture it was used as positive control.

The MTS results for HUVECs culture (Fig. 9) show a similar tendency. In this case the values obtained were independent of the treatment up to 1 day: absorbance levels did not increase significantly in any sample. Afterwards, HUVECs showed increased viability significantly on PEA surfaces and less on PEA-4d, whereas no statistically significant absorbance increase was observed for P(EA-co-AAc). 
After staining with haematoxylin-eosin, slight differences between samples were observed. In general, better results were found with fibroblasts (Fig. 8) than with endothelial cells. Fibroblasts viability was suitable, and even several layers of cells were arranged on specific areas of the surfaces after 5 days of culture. A very good initial adhesion was also observed for P(EA-co-AAc). Despite the better results obtained in the MTS assay with control wells, the stained cells shape and density suggests that the investigated materials induce a better initial adhesion than the glass cover, and cell viability on the treated PEA or the copolymer does not seem to be worse.

Haematoxylin-eosin staining images of HUVECs (Fig. 9) show a better initial attachment on PEA as compared to the other polymers; nonetheless, after 5 days cells had properly proliferated and exhibited an elongated morphology on all studied substrates. At low magnification, it can be observed that cells tend to form scattered circular structures from the first time point, which is only few hours after the seeding, and grow into more complex structures, formed by more cells in this circular disposition contacting between them with time.

\section{Discussion}

The PEA matrix polymer of scaffolds prepared as in $[9,10,25]$ suffers a surface modification as a consequence of the template rinsing process. In order to characterize these modifications, PEA was exposed to nitric acid aqueous solutions for different times, and compared with PAAc and a copolymer. This has allowed to identify the physico-chemical changes produced and to quantify them. 
The exposure of PEA polymer to nitric acid aqueous solutions seemed to change a number of ethyl acrylate monomer units into ethyl acrylate ones, by replacement of a hydrogen for the ethyl group, thus reverting the esterification process by which ethyl acrylate monomer results from acrylic acid [27].

The emergence of new bands on the FTIR spectra (Fig. 2-A) at 3500 and $3200 \mathrm{~cm}^{-1}$ agrees with such substitution. The different behaviour exhibited by untreated and acidtreated samples when swollen in water or in a basic aqueous medium ( $\mathrm{NaOH} 0.2 \mathrm{M})$ (Fig. 2-A), indicates that the material becomes more hydrophilic and, more importantly, $\mathrm{pH}$ sensitive when treated with $\mathrm{HNO}_{3}$. The fact that a dramatic increase of the $W C$ occurs at basic $\mathrm{pH}$ for the acid-treated acrylates, proportional to the immersion time, as happens in the control polymers containing acid side groups PAAc and P(EA-co-AAc) (Fig. 3-B), confirms the presence of acid groups in the treated samples, which are dissociated in basic media turning polymers into superabsorbents [28]. This gradual modification was visually supported by toluidine blue staining (specific for acid groups) (Fig. 7). The darker and more bluish samples were those where carboxylic acid groups were introduced, either by the acid treatment or by the co-polymerization with AAc.

Considering the results obtained with different techniques, it can be stated that the behaviour of PEA-4d and PEA-9d is similar to that of the 90:10 P(EA-Co-AAc) copolymer, as the last exhibited an intermediate behaviour between them. PEA-9d attained the same equilibrium values and followed the same trend as P(EA-co-AAc) in the swelling study (Fig. 3-B) at all $\mathrm{pH}$ values; moreover, the PEA-4d dynamicmechanical main relaxation (Fig. 6) is very close to that of $\mathrm{P}(\mathrm{EA}-\mathrm{co}-\mathrm{AAc})$. But it is PEA-9d whose degradation thermogram resembles more that of the copolymer (Fig. 4- 
B). Visually, with the toluidine blue staining, the colouring of P(EA-co-AAc) lies between those of PEA-4d and PEA-9d (Fig. 6).

The PEA-17d exhibited an erratic behaviour. For instance, in the swelling study (Fig. 3B), it reached the highest values (except for the PAAc) outside the trend, independently from the $\mathrm{pH}$ value, while its density (Fig.4-A) was again below the PAAc density but greater than those of the other materials. Its glass transition (Fig. 5) and $\tan \delta$ maximum temperature (Fig. 6) were close to those of PAAc. Despite the fact that the thermal degradation profiles of PEA-17d and PAAc are very different (Fig. 4-B), the percentages of solid residue left are very similar. This anomalous performance suggests that such a long treatment in nitric acid induces other chemical modifications besides the random hydrolysis of side chains, which would require a further characterization.

The nitric acid treatment has an effect on the PEA surface properties and therefore on the wettability. The water contact angle (Fig. 7) measured in the dry state experienced scarce changes with the increase of the acid treatment duration, and surprisingly the copolymer with only a small fraction of AAc groups showed a lower contact angle than PAAc. This can be attributed to the acid side groups that reduce chain mobility, leading to a vitrification of the materials at room temperature, which leads to a poorer exposure of hydroxyl groups at the surface. On the contrary, the plasticising effect of water improves chain mobility; thus contact angle values exhibited the expected trend when the materials were in their swollen states, with a proportional reduction of the angle with the immersion time in acid or the increase of AAc units. The exception was, once more, PEA 17-d. 
If it is assumed that the acid treatment affects PEA only by transforming ethyl acrylate units into acrylic acid units, the density and DSC results combined with mathematical models can be used to estimate the fraction of each component in the treated materials. With the measured densities and considering the mixture of both homopolymers as ideal, for which: $\frac{1}{\rho}=\frac{1}{\rho_{1}} \cdot \omega_{1}+\frac{1}{\rho_{2}} \cdot \omega_{2}$, where $\rho$ is the density of the mixture, $\omega_{1}$ and $\omega_{2}$ are the mass fractions of components 1 and 2, and $\rho_{1}$ and $\rho_{2}$ are their densities, the fraction of each component in the treated PEA was estimated. Firstly, it was verified for the copolymer (whose composition was known) that the ideal mixture was a good model for this system (its measured density was $1.158 \mathrm{~g} / \mathrm{cm}^{3}$ and the calculated one was $1.148 \mathrm{~g} / \mathrm{cm}^{3}$, which was considered close enough). For PEA-4d, a fraction of acrylic acid units, $\omega_{2}$, of 0.09 was obtained, 0.18 for PEA-9d and 0.32 for PEA-17d.

It is also possible to determine the fraction of each component in the mixture from its glass transition temperature, $T_{\text {gmix }}$, those of the pure homopolymers PEA and PAAc $\left(T_{g 1}, T_{g 2}\right)$, and their heat capacity jumps at $\operatorname{Tg}\left(\Delta C_{p 1}, \Delta C_{p 2}\right)$ by using CouchmanKarasz’s expression [29]:

$$
\ln \left(T_{g \text { mix }}\right)=\omega_{1} \cdot \Delta C_{p 1} \cdot \ln \left(T_{g 1}\right)+\omega_{2} \cdot \Delta C_{p 2} \cdot \ln \left(T_{g 2}\right) \Delta C_{p 1} \cdot \omega_{1}+\Delta C_{p 2} \cdot \omega_{2}
$$

If the ratio $T_{g P E A} / T_{g P A A c}$ is close to 1 , which is here the case, this expression can be simplified by removing the logarithms to:

$$
T_{g \text { mix }}=\frac{\omega_{1} \cdot \Delta C_{p 1} \cdot T_{g 1}+\omega_{2} \cdot \Delta C_{p 2} \cdot T_{g 2}}{\Delta C_{p 1} \cdot \omega_{1}+\Delta C_{p 2} \cdot \omega W_{2}}
$$


With the DSC results and the Couchman-Karasz simplified expression, findings quite different to the previous ones were obtained. For PEA-4d the estimated acrylic acid fraction was 0.27 , for PEA-9d it was 0.44 , and for PEA-17d a value of 0.87 was obtained. These discrepancies with the values predicted from densities could be attributable to the low $T_{g}$ measured for PAAc, as compared to values previously reported [30].

The biological experiments show that the modification of the PEA does not compromise its use as a scaffold: an increase in hydrophilicity of the scaffold improves the seeding of cells and promotes cell migration towards the interior. In fibroblasts cultures on films, for all times, PEA, PEA-4d and P(EA-co-AAc) showed a great density of cells, even sometimes higher than on the control, with extended cell morphology (Fig.8). Previous works reported that substrates containing small fractions of carboxylic acid groups enhanced keratocytes attachment and proliferation [31]; in other works, their presence led to greater densities of fibronectin or oriented it with more accessible binding sites, what enhanced cell attachment [32].

We could attribute the better outcome of PEA over P(EA-co-AAc) or PEA-4d to an initial variation of the $\mathrm{pH}$ medium, because of the presence of side acid groups, that might affect cells metabolism reducing absorbance levels. The reason for the stained samples showing a greater density than anticipated by MTS results could be found in the volume of medium in which they were cultured: $200 \mu \mathrm{L}$ for the samples employed for MTS assays, whereas $400 \mu \mathrm{L}$ for the stained samples. Probably, this increase of medium was enough to buffer the $\mathrm{pH}$ variation induced by the acid groups of the samples. 
HUVECs are very sensitive cells, and nonetheless they showed to be viable and assembled into circular structures on all the materials (Fig. 9-C,F,I). The good outcome could be favored by the conditioning step in medium containing FBS; indeed, previous works reported that the presence of $\mathrm{COOH}$ groups enhanced the deposition of fibronectin and albumin encouraging endothelial cells growth [33]. Analogously, works report that human mesenchymal stem cells (hMSCs) can grow on substrates containing acrylic acid units after being coated with collagen [20,34]. These positive results open the possibility to obtaining vascular-like structures when HUVECs are seeded in these three-dimensional PEA scaffolds.

\section{Conclusions}

Immersion of PEA in nitric acid aqueous solutions, as is employed for scaffolds production, transforms the homopolymer into a random copolymer of ethyl acrylate and acrylic acid, with a proportion of both that depends on the immersion time. By comparing the physico-chemical properties of PEA with different times of exposure with those of a P(EA-co-AAc) copolymer it was concluded that the weight fraction of monomer units converted was about $10 \%$ after 4 days. As a consequence of these changes, PEA becomes more hydrophilic, exhibits $\mathrm{pH}$-dependent swelling, and is capable of vitrifying at physiological temperature in the dry state. Nevertheless, the chemical modifications occurred at these times do not impair the biological performance of the material, which continues to be excellent even with very sensitive cells. If immersion in nitric acid solution lasts longer, other chemical changes that have not been identified might take place. 


\section{Acknowledgements}

The authors acknowledge financing through project FP7 NMP3-SL-2009-229239 "Regeneration of cardiac tissue assisted by bioactive implants" (RECATABI) and projects MAT2011-28791-C03-02 and -03 from the Spanish Ministerio de Ciencia e Innovación. This work was also supported by the Spanish Ministry of Education through M. Arnal-Pastor FPU AP2009-1870 grant. 


\section{References}

[1] A.J Campillo-Fernández, R.E Unger, K. Peters, S. Halstenberg, M. Santos, Salmerón Sánchez M, et al., Analysis of the biological response of endothelial and fibroblast cells cultured on synthetic scaffolds with various hydrophilic/hydrophobic ratios. Influence of fibronectin adsorption and conformation, Tissue Eng. 15 (2009) $1331-1341$.

[2] P. Rico, J.C. Rodríguez Hernández, D. Moratal, M. Monleón Pradas, M. Salmerón Sánchez, Substrate-induced assembly of fibronectin into networks. Influence of surface chemistry and effect on osteoblast adhesion, Tissue Eng. 15 (2009) 3271-3281.

[3] J.M. Soria, M. Sancho-Tello, M.A. García Esparza, V. Mirabet, J.V. Bagan, M. Monleón, et al., Biomaterials coated by dental pulp cells as substrate for neural stem cell differentiation, J Biomed Mater Res Part A. 97 (2011) 85-92.

[4] J.M. Soria, C. Martínez-Ramos, V. Benavent, A. Campillo-Fernández, J.L. GómezRibelles, M. Salmerón-Sánchez, et al., Survival and differentiation of embryonic neural explants onto different biomaterials, J Biomed Mater Res Part A. 79 (2006) 495-502.

[5] A.J. Campillo-Fernández, S. Pastor, M. Abad-Collado, L. Bataille, J.L. Gómez Ribelles, J.M. Meseguer Dueñas, et al., Future design of a new keratoprosthesis. Physical and biological analysis of polymeric substrates for epithelial cell growth, Biomacromolecules. 8 (2007) 2429-2436.

[6] M. Pérez Olmedilla, N. García-Giralt, M. Monleón Pradas, P. Benito Ruiz, J.L. Gómez Ribelles, E. Cáceres Palou, et al., Response of human chondrocytes to a nonuniform distribution of hydrophilic domains on poly(ethyl acrylate-co-hydroxyethyl methacrylate) copolymers, Biomaterials. 27 (2006) 1003-1012. 
[7] M. Ying, K. Saha, S.R. Bogatyrev, J. Yang, A.L. Hook, Z.I. Kalcioglu, et al., Combinatorial development of biomaterials for clonal growth of human pluripotent stem cells, Nature Materials. 9 (2010) 768-778.

[8] J.C. Chachques, M. Monleón Pradas, A. Bayés-Genís, C. Semino, Creating the bioartificial myocardium for cardiac repair: challenges and clinical targets, Expert Rev. Cardiovasc. Ther. 11 (2013) 1701-1711.

[9] R. Brígido Diego, M. Pérez Olmedilla, A. Serrano Aroca, J.L. Gómez Ribelles, M. Monleón Pradas, G. Gallego Ferrer, M. Salmerón Sánchez, Acrylic scaffolds with interconnected spherical pores and controlled hydrophilicity for tissue engineering, J.Mater. Sci.: Mater. in Medicine. 16 (2005) 693-698.

[10] C. Martínez-Ramos, A. Vallés-Lluch, J.M. García Verdugo, J.L. Gómez Ribelles, J.A. Barcia Albacar, A. Baiget Orts, et al., Channeled scaffolds implanted in adult rat brain, J Biomed Mater Res A. 100 (2012) 3276-3286.

[11] A. Vallés-Lluch, M. Arnal-Pastor, M. Keicher, M. Monleón Pradas, Coating typologies and constrained swelling of hyaluronic acid gels within scaffold pores, $\mathrm{J}$ Colloid Interface Sci. 361 (2011) 361-369.

[12] B.G. Keselowsky, D.M. Collard, A.J. García, Surface chemistry modulates focal adhesion composition and signaling through changes in integrin binding, Biomaterials. 25 (2004) 5947-54.

[13] J.Z. Luk, J. Cork, J. Cooper-White, L. Grøndahl, Use of two-step grafting to fabricate dual-functional films and site-specific functionalized scaffolds, Langmuir. 31 (2015) 1746-54.

[14] H. Takahashi, N. Matsuzaka, M. Nakayama, A. Kikuchi, M. Yamato, T. Okano, Terminally functionalized thermoresponsive polymer brushes for simultaneously promoting cell adhesion and cell sheet harvest, Biomacromolecules. 13 (2012) 253-60. 
[15] V. Gubala, N.C. Le, R.P. Gandhiraman, C. Coyle, S. Daniels, D.E. Williams, Functionalization of cyclo-olefin polymer substrates by plasma oxidation: stable film containing carboxylic acid groups for capturing biorecognition elements, Colloids Surf B Biointerfaces. 81 (2010) 544-8.

[16] V. Llopis-Hernández, P. Rico, J. Ballester-Beltrán, D. Moratal, M. SalmerónSánchez, Role of surface chemistry in protein remodeling at the cell-material interface, PLoS One. 6 (2011) e19610.

[17] J. Wu, K. Xue, H. Li, J. Sun, K. Liu, Improvement of PHBV scaffolds with bioglass for cartilage tissue engineering, PLoS One. 2013 Aug 9;8(8):e71563.

[18] B.G. Keselowsky, D.M. Collard, A.J. García, Surface chemistry modulates focal adhesion composition and signaling through changes in integrin binding, Biomaterials. 25 (2004) 5947-54.

[19] M. Buttiglione, F. Vitiello, E. Sardella, L. Petrone, M. Nardulli, P. Favia, R. d'Agostino, R. Gristina, Behaviour of SH-SY5Y neuroblastoma cell line grown in different media and on different chemically modified substrates, Biomaterials. 28 (2007) 2932-45.

[20] N.M. Coelho, C. González-García, J.A. Planell, M. Salmerón-Sánchez, G. Altankov, Different assembly of type IV collagen on hydrophilic and hydrophobic substrata alters endothelial cells interaction, Eur Cell Mater. 19 (2010) 262-72.

[21] A. Vallés Lluch, M. Arnal-Pastor, C. Martínez-Ramos, G. Vilariño-Feltrer, L. Vikingsson, C. Castells-Sala, C.E. Semino, M. Monleón Pradas, Combining selfassembling peptide gels with three-dimensional elastomer scaffolds, Acta Biomaterialia 9 (2013) 9451-60.

[22] C. Martínez-Ramos, E. Rodríguez-Pérez, M.P. Garnes, J.C. Chachques, D. Moratal, A. Vallés Lluch, M. Monleón Pradas, Design and assembly procedures for 
large-sized biohybrid scaffolds as patches for myocardial infarct, Tissue Engineering, Part C: Methods 20 (2014) 817-27.

[23] C. Castells-Sala, A. Vallés-Lluch, C. Soler-Botija, M. Arnal-Pastor, C. MartínezRamos, T. Fernandez-Muiños, N. Marí-Buyé, A. Llucià-Valldeperas, B. Sanchez, J.C. Chachques, A. Bayes-Genis, M. Monleón Pradas, C.E. Semino, Development of bioactive patch for maintenance of implanted cells at the myocardial infarcted site, Journal of Nanomaterials 2015; article ID 804017.

[24] J. Más Estellés, I. Krakovsky, J.C. Rodríguez Hernández, A.M. Piotrowska, M. Monleón Pradas, Mechanical properties of porous crosslinked poly(ethyl-acrylate) for tissue engineering, J. Mater. Sci. 42 (2007) 8629-8635.

[25] J.C. Rodríguez Hernández, A. Serrano Aroca, J.L. Gómez Ribelles, M. Monleón Pradas, Three-dimensional nanocomposite scaffolds with ordered cylindrical orthogonal pores, J Biomed Mater Res B: Appl Biomaterials. 84 (2008) 541-549.

[26] P. J. Elving , J. M. Markowitz , Isadore. Rosenthal, Preparation of Buffer Systems of Constant Ionic Strength, Anal. Chem. 28 (7) (1956) 1179-1180.

[27] T. Ohara, T. Sato, N. Shimizu, G. Prescher, H. Schwind, O. Weiberg, K. Marten, H. Greim, Acrylic Acid and Derivatives. In: Ullmann's Encyclopedia of Industrial Chemistry, Wiley-VCH Verlag, Weinheim 2012; pp 1-19.

[28] E.H. Rifi, F. Rastegar, J.P. Brunette, Uptake of cesium, strontium and europium by a poly(sodium acrylate-acrylic acid) hydrogel, Talanta. 42 (1995) 811-816.

[29] P.R. Couchman, Compositional Variation of Glass-Transition Temperatures. Application of the Thermodynamic Theory to Compatible Polymer Blend, Macromolecules 11 (1978) 1156-1161. 
[30] A. Eisenberg, T. Yokoyama, E. Sambalido, Dehydration kinetics and glass transition of poly(acrylic acid), Journal of Polymer Science Part A-1: Polymer Chemistry. 7 (1969) 1717-1728.

[31] D.B. Haddow, R.M. France, R.D. Short, S. MacNeil, R.A. Dawson, G.J. Leggett, E. Cooper, Comparison of proliferation and growth of human keratinocytes on plasma copolymers of acrylic acid/1,7-octadiene and self-assembled monolayers, J Biomed Mater Res. 47 (1999) 379-87.

[32] C.A. Scotchford, C.P. Gilmore, E. Cooper, G.J. Leggett, S. Downes, Protein adsorption and human osteoblast-like cell attachment and growth on alkylthiol on gold self-assembled monolayers, J Biomed Mater Res. 59 (2002) 84-99.

[33] D.T. Caren, I. Sylvie, D.R. Buddy, J.T. Barbara, A. Sundar, D. Allara, Endothelial Cell Growth and Protein Adsorption on Terminally Functionalized, Self-Assembled Monolayers of Alkanethiolates on Gold, Langmuir. 13 (1997) 3404-3413

[34] B. Gupta, C. Plummer, I. Bisson, P. Frey, J. Hilborn, Plasma-induced graft polymerization of acrylic acid onto poly(ethylene terephthalate) films: characterization and human smooth muscle cell growth on grafted films, Biomaterials. 23 (2002) 86371. 


\section{Figure captions}
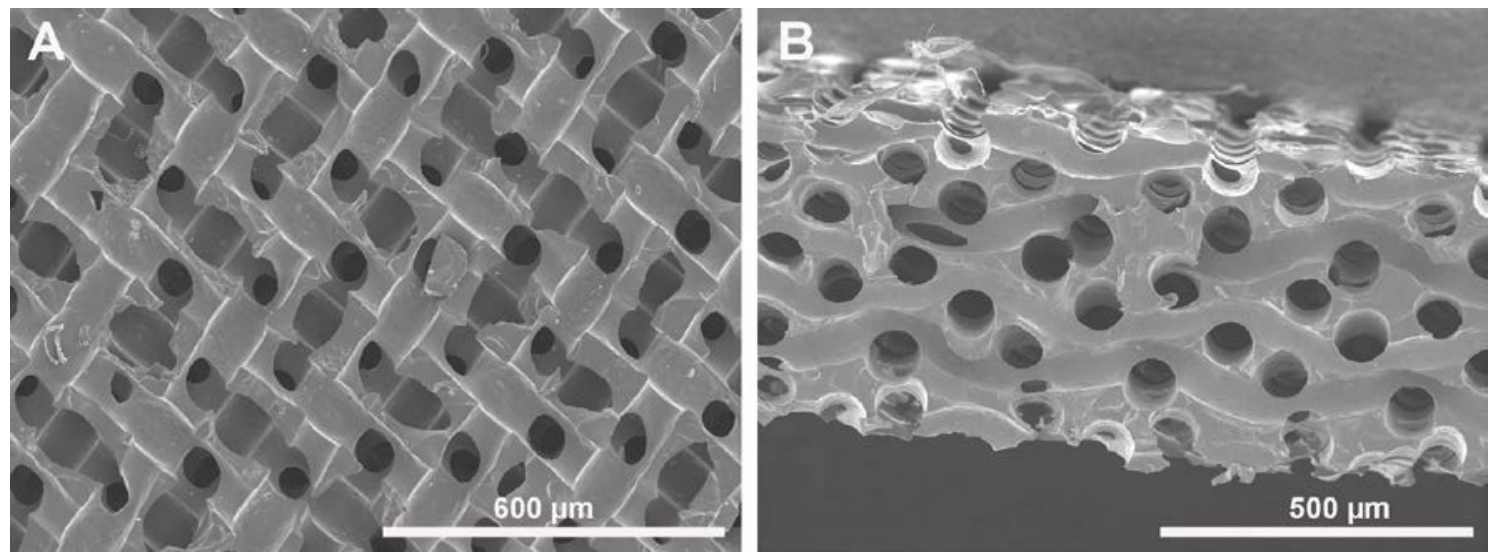

Figure 1. SEM images of the PEA scaffolds with cylindrical orthogonal pores: (A) surface, (B) cross-section. Scale bare: $600 \mu \mathrm{m}$ (A) and $500 \mu \mathrm{m}$ (B)

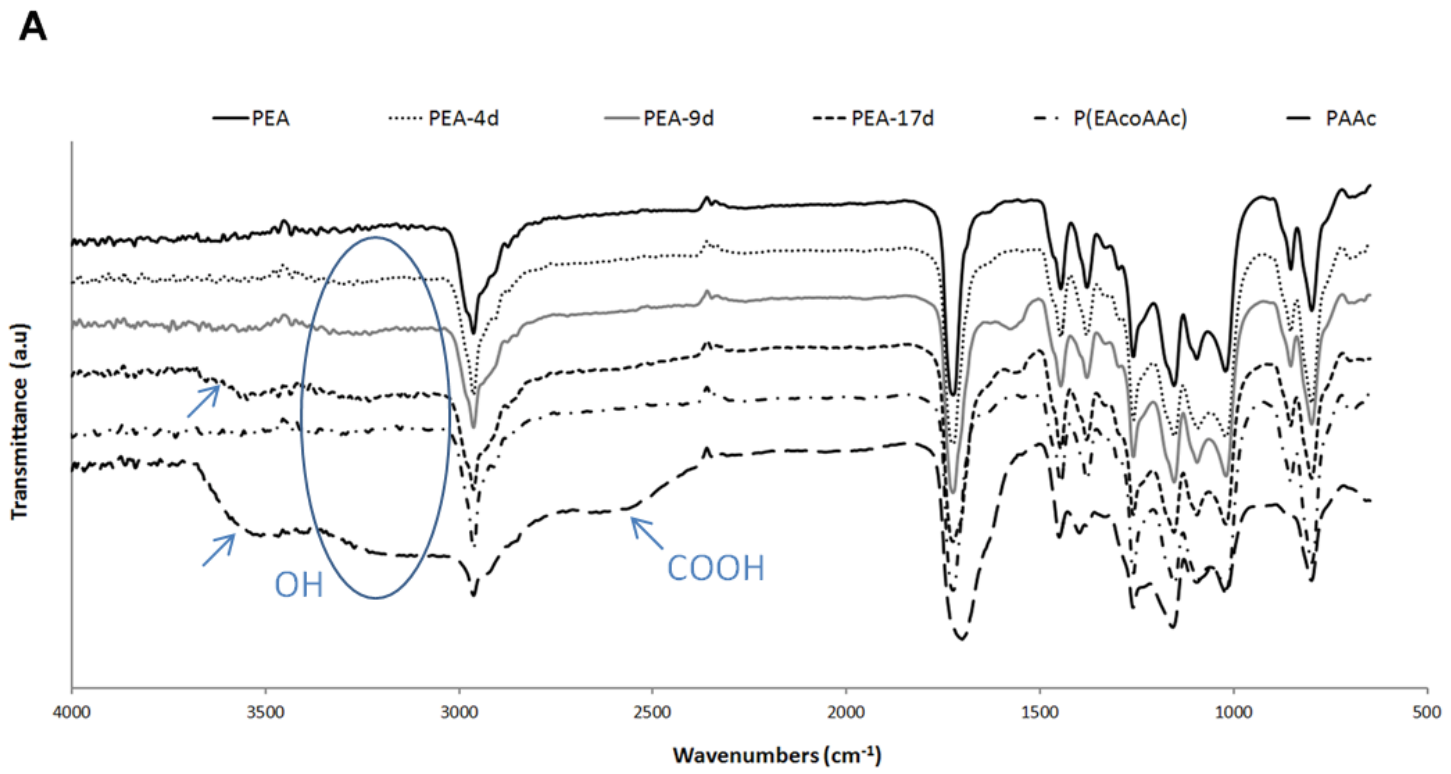

B

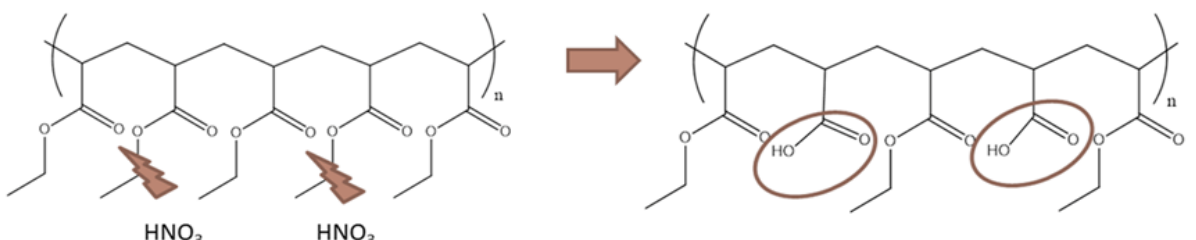

Figure 2. (A) FTIR spectra of PEA, PEA treated for 4, 9 and 17 days in nitric acid, PAAc and the copolymer P(EA-co-AAc), in the 500 to $4000 \mathrm{~cm}-1$ region. (B) Scheme of the reaction taking place during the HNO3 treatment 


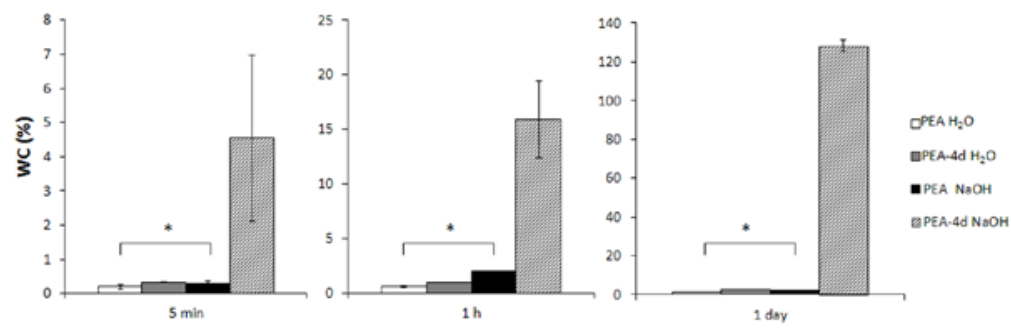

B

C

D

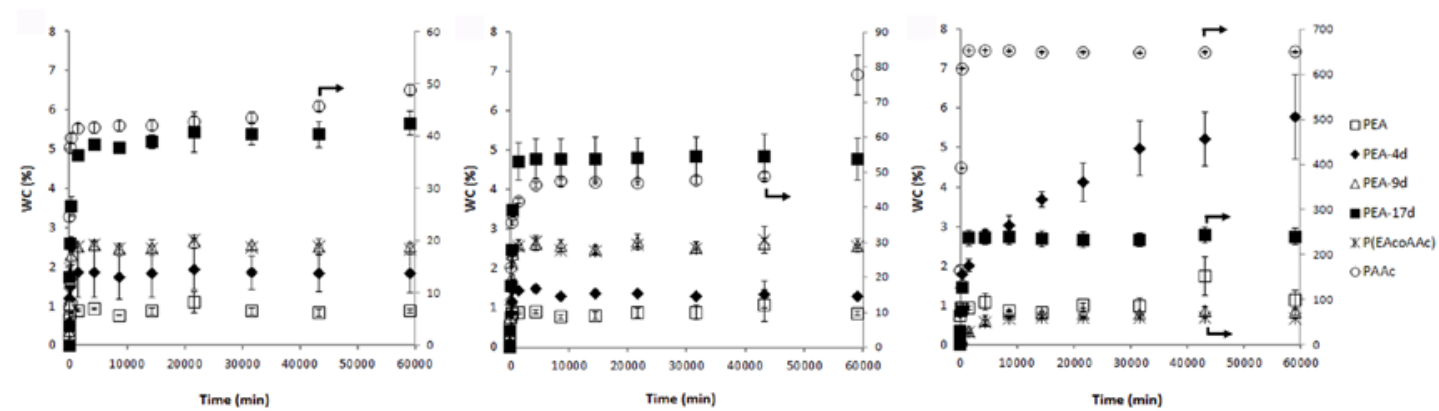

Figure 3. (A) Water content of the untreated and 4 days acid-treated PEA samples as a function of time of immersion in water or in a $\mathrm{NaOH} 0.2 \mathrm{M}$ aqueous solution. (*) No significant differences were observed between PEA swollen in water or NaOH (aq) for all time points. (B-D) Water content WC of acid-treated PEA samples and of P(EA-coAAc) and PAAc as a function of time of immersion in solutions with pH equal to 2 (B), 4.6 (C) and 8 (D). Arrows indicate the data series to be read with the right-hand scale on the plots. For PEA, no significant differences were observed at different pH values. For the PEA-4d, PEA-9d, PEA-17d and P(EA-co-AAc) no significant differences were observed between the $E W C$ attained at $\mathrm{pH}=2$ and $\mathrm{pH}=4.6$. 
A

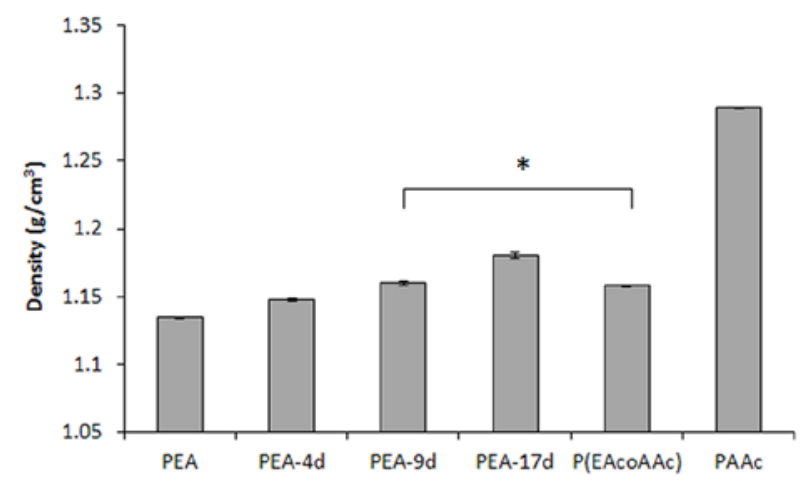

B

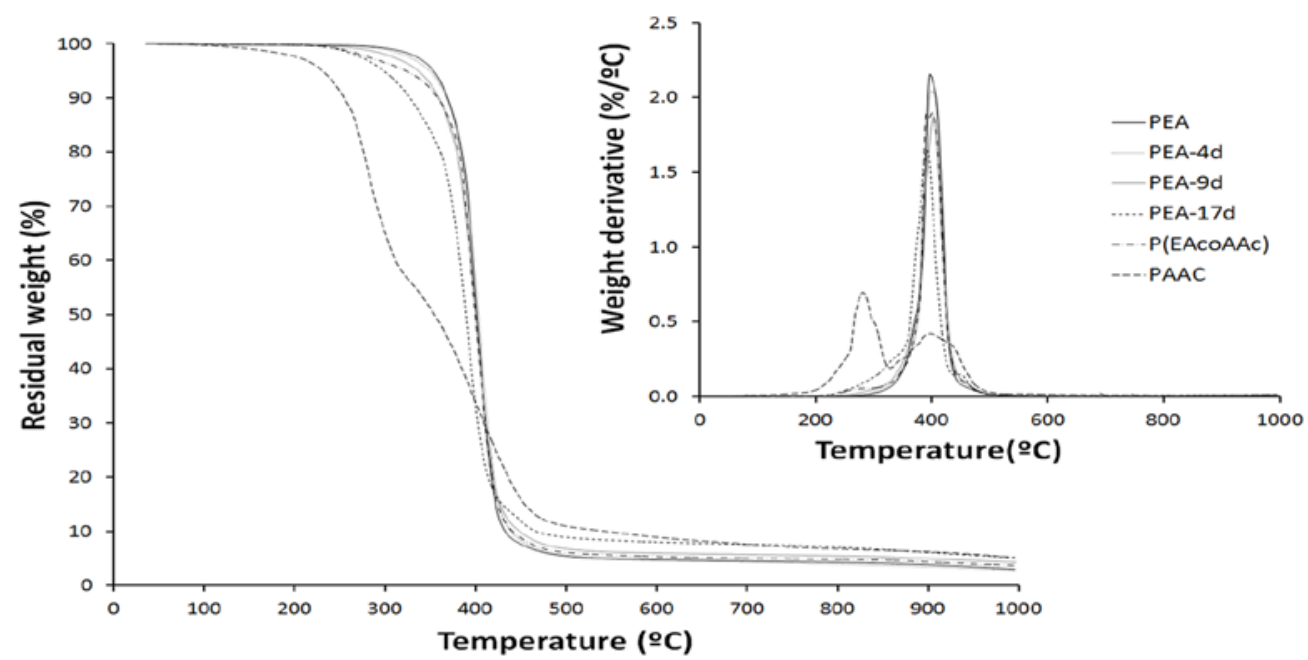

Figure 4. (A) Densities of acid-treated PEA samples and P(EA-co-AAc) and PAAc references. (*) No significant differences were observed between the PEA-9d and P(EA-co-AAc) densities. (B) Thermograms for acid-treated PEA, P(EA-co-AAc) and PAAc samples, plotting the residual mass fraction as a function of temperature, and the first derivative of the residual mass fraction as a function of temperature (inset). 


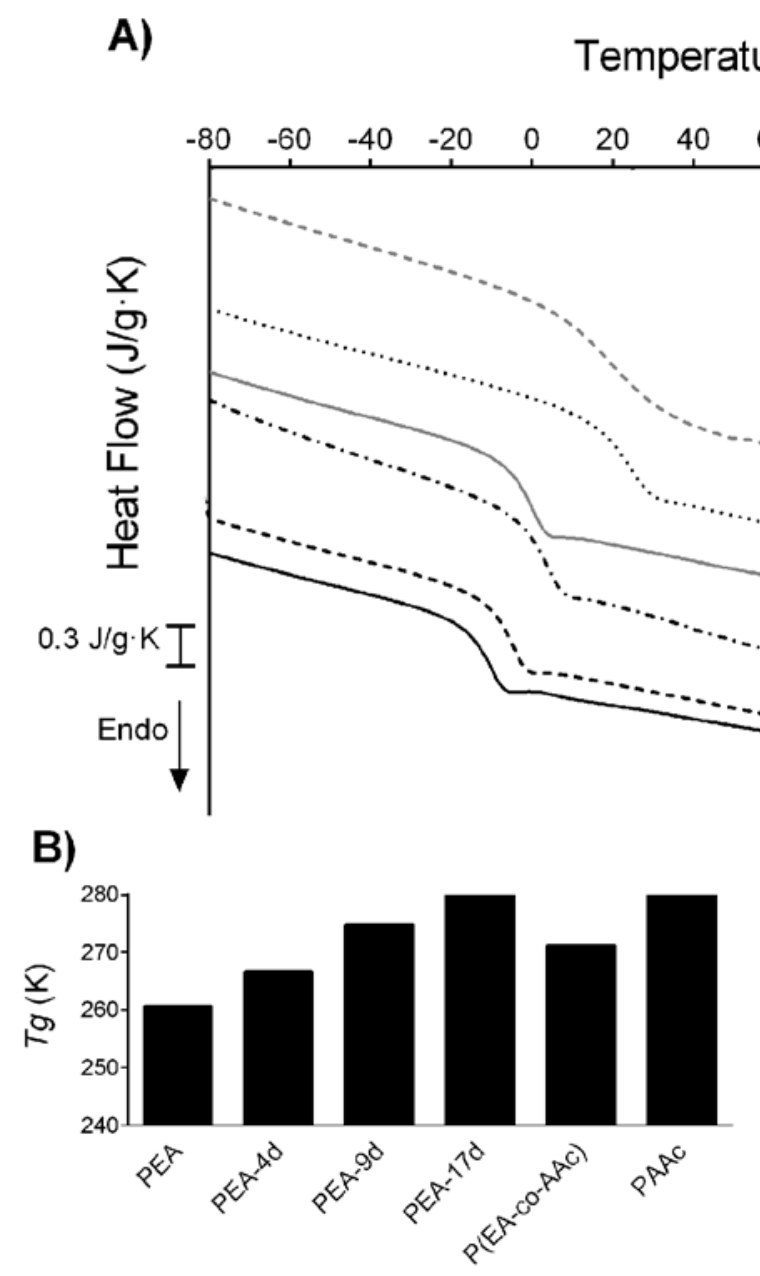

\section{C)}

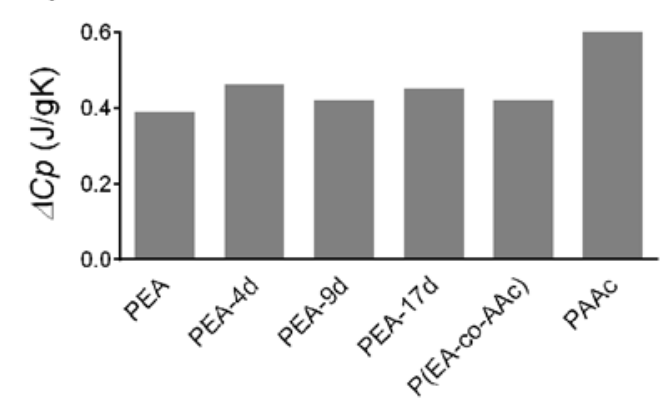

Figure 5. (A) DSC thermograms plotting the heat flux per unit mass and unit temperature as a function of temperature of acid-treated PEA samples and the P(EA-coAAc) and PAAc references. (B) Glass transition temperature and (C) specific heat capacity jump at the glass transition for the different materials. 

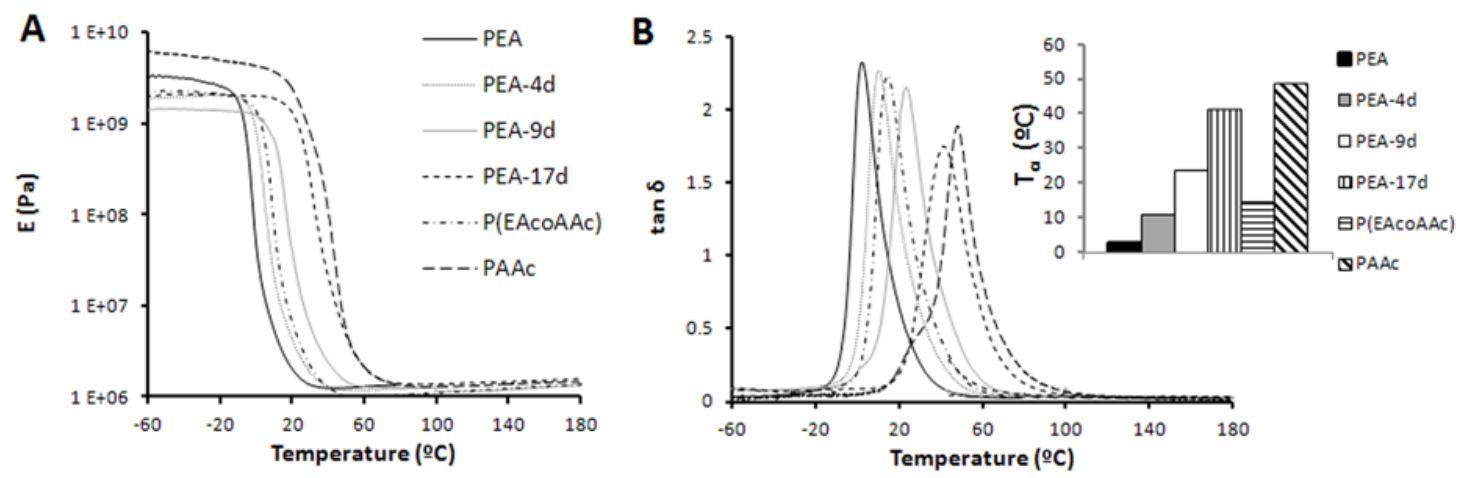

Figure 6. Elastic modulus variation with temperature (A) for acid-treated PEA samples and $\mathrm{P}(\mathrm{EA}-\mathrm{co}-\mathrm{AAc})$ and PAAc references, $\tan \delta$ as a function of temperature (B), and $\mathrm{T}_{\alpha}$ (inset).
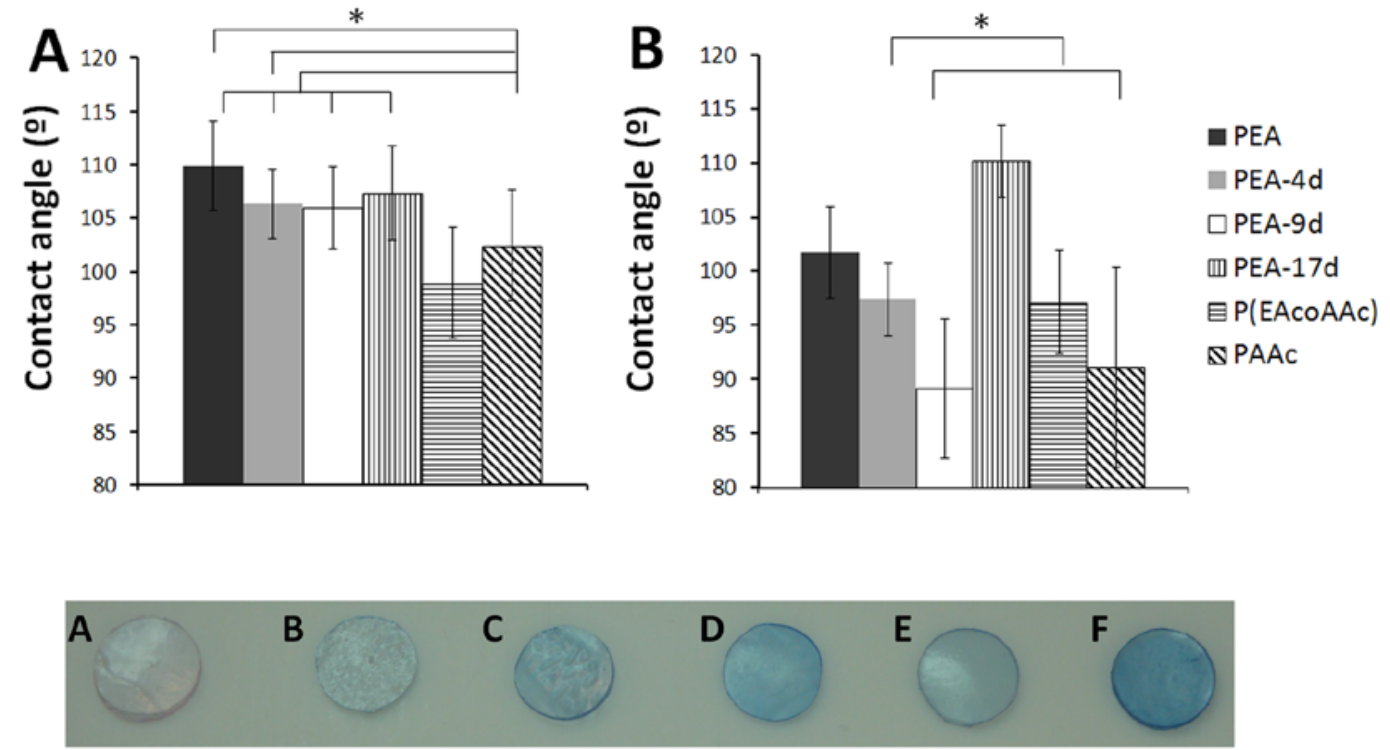

Figure 7. Water contact angle measurements of dry (A) and swollen (B) acid-treated PEA, P(EA-co-AAc) and PAAc samples. (*) No statistically significant differences were observed between the labelled materials. (A-F) Toluidine staining for PEA (A), PEA-4d (B), PEA-9d (C) PEA-17d (D), P(EA-co-AAc) (E), and PAAc (F). 


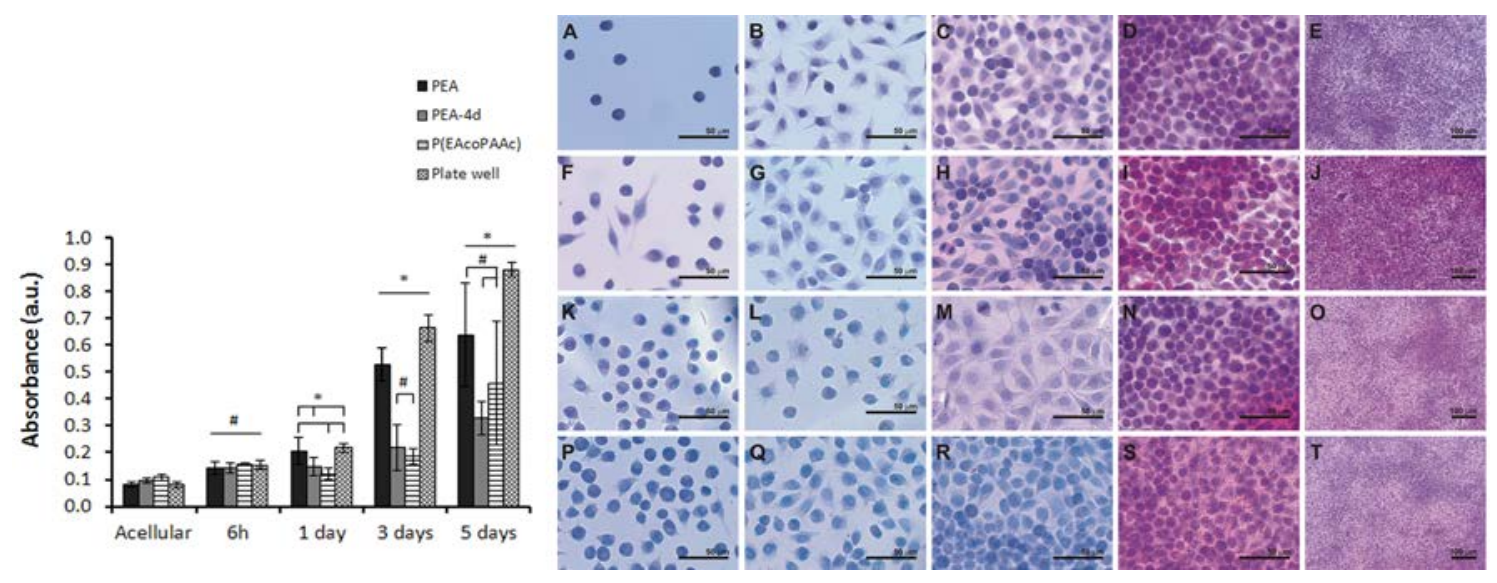

Figure 8. MTS results after 6h, 1, 3 and 5 days culture of L929 fibroblasts on untreated PEA, PEA-4d, P(EA-Co-AAc) and plate wells. Bright field images of samples stained with haematoxylin-eosin after (from left to right) 6h, 1, 3 and 5 days at $40 \mathrm{X}$ magnification, and 5 days at 10X magnification, after culture of L929 fibroblasts on covers (A,B,C,D,E), PEA (F,G,H,I,J), PEA-4d (K,L,M,N,O) and P(EA-co-AAc) (P,Q,R,S,T). (*) Differences are statistically significant. (\#) Differences are not statistically significant.

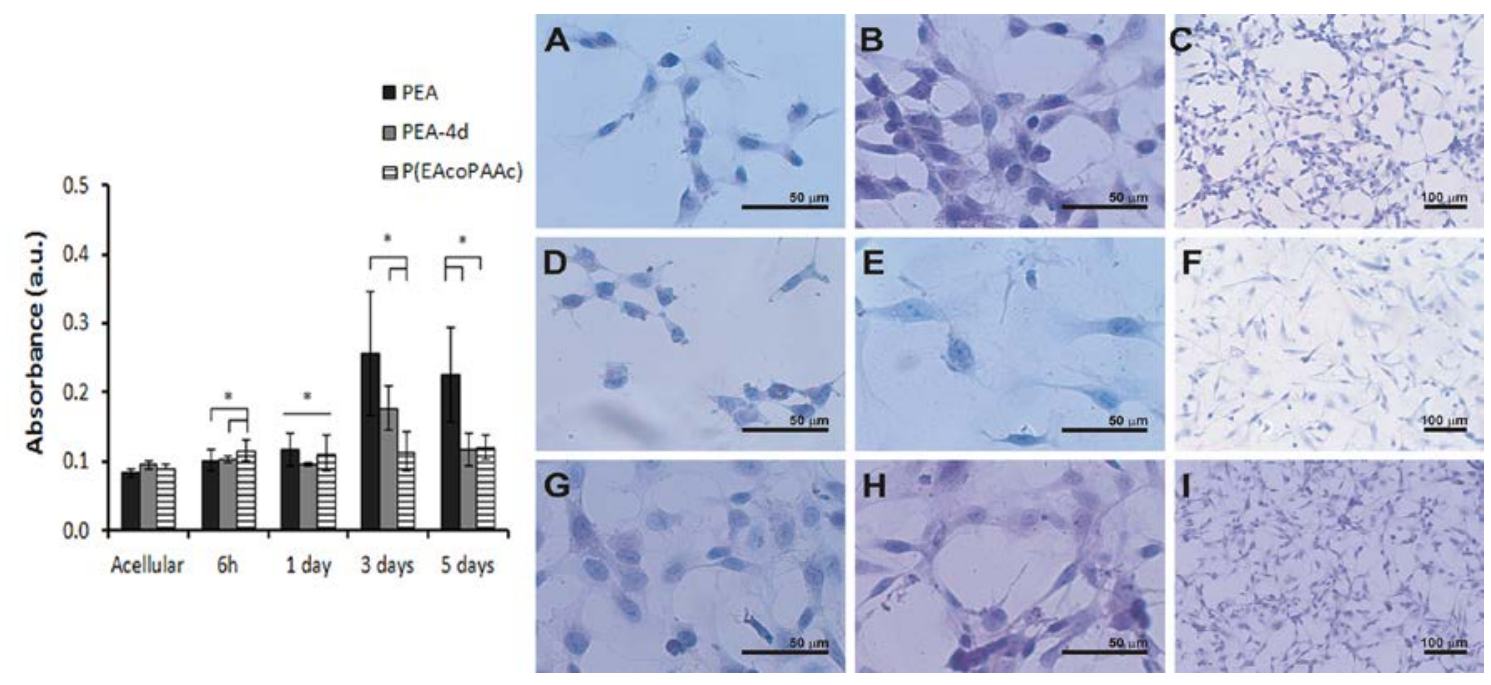

Figure 9. MTS results after 6h, 1, 3 and 5 days culture of HUVECs on untreated PEA, PEA-4d, P(EA-co-AAc) and plate wells. Bright field images of samples stained with haematoxylin-eosin after (from left to right) 6h, and 5 days at $40 \mathrm{X}$ magnification and after 5 days at 10X magnification of HUVECs culture on PEA (A,B,C), PEA-4d 
(D,E,F) and P(EA-co-AAc) (G,H,I). (*) Differences are statistically significant. (\#) Differences are not statistically significant. 